\title{
SELECTIVE RADIATION FROM VARIOUS SOLIDS II.
}

\author{
By W. W. Coblentz.
}

While it is evidently important to improve upon the accuracy of the results already at hand by reinvestigating the known emissive properties of matter, it is equally important to investigate unknown substances. Believing that only through an immense amount of routine work will any marked advance be made in our knowledge of the laws governing the spectral partition of energy at various temperatures, it is intended from time to time to examine and place on record the behavior of representative examples of various groups of chemical compounds. Many of these substances will probably need no further investigation, but there are already a considerable number known, having unusual emission spectra, which will deserve a detailed investigation at some future time. The immediate application (particularly the commercial application) may not always be apparent, just as was the investigation of the reflecting power of metals made some six years ago, which until recently has had but little application.

In previous papers ${ }^{1}$ attention was called to the fact that the intensities of discrete spectral lines increases in proportion to the energy input, i. e., with the temperature, while in the case of the solids investigated, which have banded emission spectra, the distribution of intensities among the emission bands varies with rise in temperature. The distribution of energy seems to follow a law similar to that of a complete radiator in which the wave length of maximum intensity decreases with rise in temperature. In other words, if we draw the envelope through the points of maximum intensity of the emission bands, then the point of maximum intensity of the envelope shifts toward the 
shorter wave lengths with rise in temperature, just as is true of substances, e. g., metals, emitting a perfectly smooth continuous spectrum. This was well demonstrated in the previous investigation of the isochromatics of the Nernst glower and certain other substances. The isochromatics were shown to have a double curvature, at the temperature at which the wave length of the maximum of the envelope coincided with the "isochromatic" wave length. This is in marked contrast with the growth of intensity of emission lines in gases, in vacuum tubes, in which the effect of (vapor) density is negligible. In the latter all the isochromatics ${ }^{2}$ have the same trend (i. e., none change in their curvature), showing that, for the range investigated, there is no shift in the wave length of maximum emission but simply a gradual increase in intensity of all the lines with rise in temperature. $^{3}$ This is corroborated by Nutting and Tugman ${ }^{4}$ by photometric comparisons of helium lines. On the other hand, the highpressure mercury lamps ${ }^{5}$ show a variation in the distribution of intensities among the emission lines, with rise in temperature, and hence in the density of the mercury vapor.

The following emissivity curves of talc illustrate to an unusual degree the gradual shift of the maximum intensity of emission bands toward the shorter wave lengths without the merging into a continuous spectrum which was observed in the Nernst glower.

\section{TALC $\left(\mathrm{H}_{2} \mathrm{Mg}_{3} \mathrm{Si}_{4} \mathrm{O}_{12}\right)$.}

\section{RADIATION FROM MINERALS.}

In Fig. I are given a series of spectral-energy curves of a layer of powdered talc rendered incandescent on a heater tube as in previous experiments. Curves $a$ and $b$ belong to a different series from the rest. The temperatures (roughly estimated) varied from $500^{\circ} \mathrm{C}$., curve $a$, to about $900^{\circ}$ in curve $e$. In Fig. 2, these same curves are shown on a larger scale. There are three sharp emission bands, at $2.42 \mu, 2.75 \mu$, and $3.35 \mu$, respectively, which deserve especial notice. In curve $a$ the band at $3.35 \mu$ is as intense as the

${ }^{2}$ Coblentz: Carnegie Publication No. 35, p. 3 I8.

${ }^{3}$ See also this Bulletin, 5 , pp. 18 1 et seq.

${ }^{4}$ Nutting and Tugman, Nature, 81, p. I89; 1909.

${ }^{5}$ Pflüger. Ann. der Phys. (4), 26, p. 789; 1908.

Küch and Retschinsky, Ann. der Phys., 20, p. 563, 1906; 22, pp. 595 and 852; 1907. 


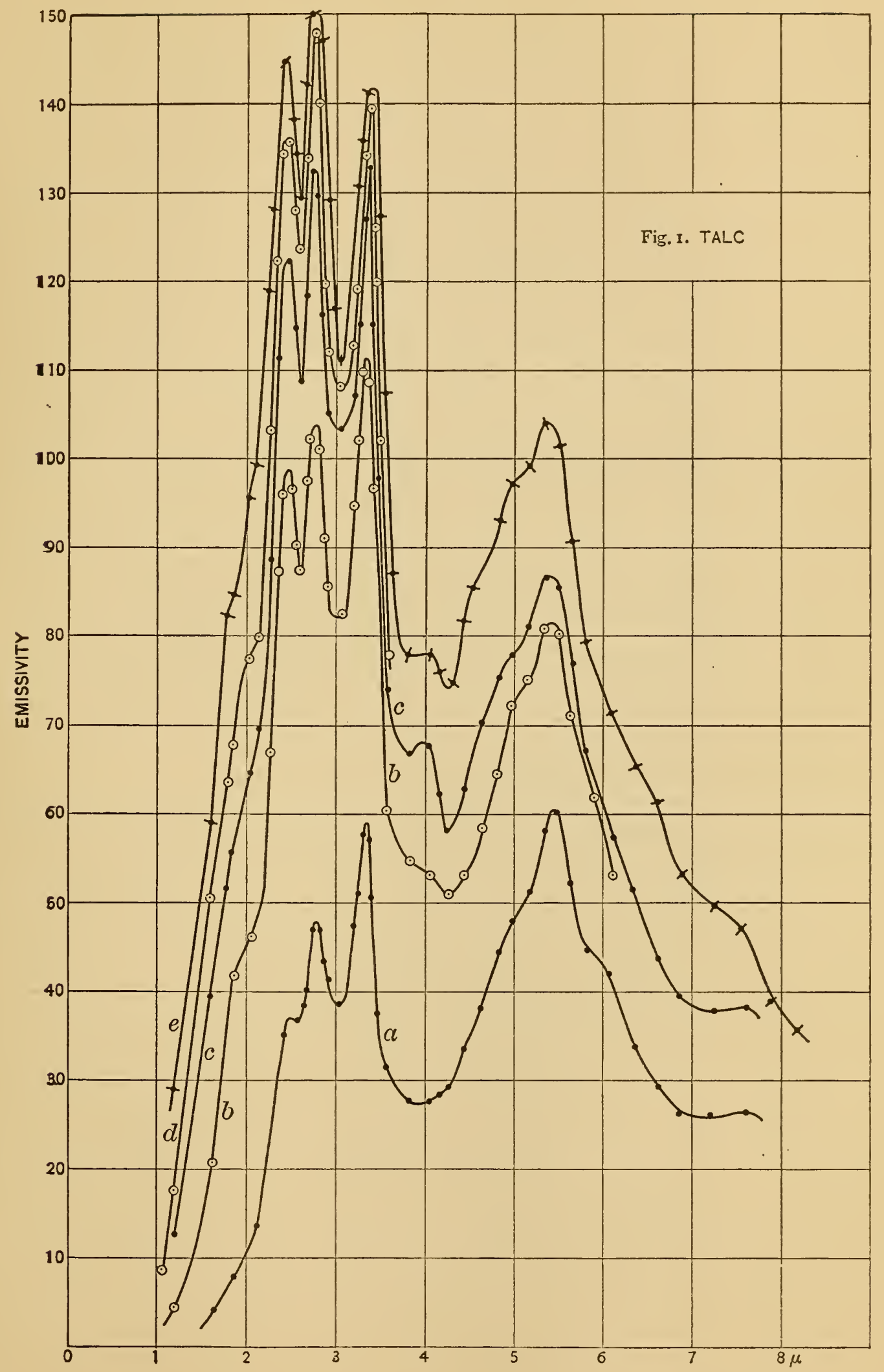


one at $5.4 \mu$, while in all of the remaining curves the spectral region at $2 \mu$ to $3 \mu$ is the more intense. In this region the band at $3.35 \mu$, curve $a$, is far more intense than those of shorter wave length, but with rise in temperature the bands at $2.42 \mu$ and $2.75 \mu$ become the most intense. In no case does the position of the maximum of the well-defined emission bands shift appreciably with rise in temperature. In Fig. 3 are given two energy curves of a rod of dehydrated talc, heated electrically as in previous experiments. The rod was made in an oxyhydrogen flame and was about $4 \mathrm{~mm}$ thick. Curve $b$ gives the spectral energy distribution of the hottest side of the rod including the bright electrically conducting streak. Curve $a$ is for the coolest side. As a result of dehydration, the maximum at $2.75 \mu$ in talc is shifted to $2.79 \mu$, the products after dehydration being the oxides of magnesium and silicon. In Fig. 3, curve $c$ is for oligoclase, previously described, with a sharp maximum at $2.87 \mu$. In Fig. 4, curves $c$ and $d$ give the spectral emission of a similar rod of dehydrated talc heated to a low red. In curve $d$ the temperature is somewhat higher, and the intersection of the two curves is due to the difference in the scale of ordinates.

\section{MAGNESIUM SILICATE $\left(\mathrm{MgSiO}_{3}\right)$.}

This is a sample of the pure material obtained from Dr. Allen, of the Geophysical Laboratory. The rod was electrically heated as in the case of the dehydrated talc. The spectra of the two substances (Fig. 4) are quite similar to magnesia; ${ }^{6}$ but in the minor details there is a marked difference, the $2.8 \mu$ silica band being weaker and the bands at $3.7,4 . \mathrm{I}$, and $4.5 \mu$ being more prominent in the pure magnesium silicate than in the dehydrated talc. The weakness of the silica band at $2.8 \mu$ is no doubt due to the fact that, in the pure silicate, the ratio of $\mathrm{MgO}$ to $\mathrm{SiO}_{2}$ is I : I, while in the dehydrated talc the proportion of silica is greater. Although these spectra seem to be the composite of the emission bands of the individual oxides, it is not generally admitted that the substances are solid solutions, but true chemical compounds. This will be noticed again on a subsequent page.

\footnotetext{
${ }^{6}$ See this Bulletin, 5, p. I69.
} 
Coblentz.]

Selective Radiation from Solids.

305
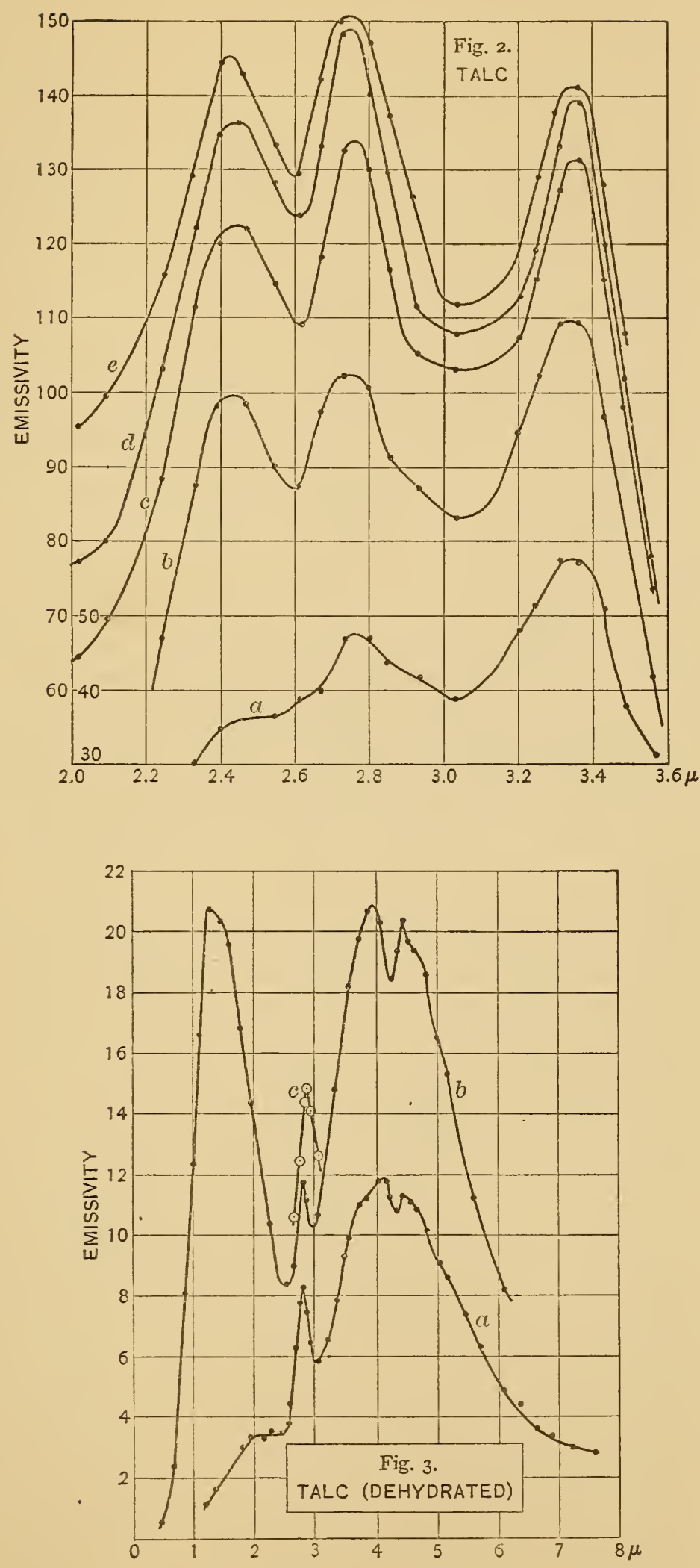
DIASPORE (A1O (OH)).

HYDROTALCITE $\left(\mathrm{Mg}_{3} \mathrm{Al}(\mathrm{OH})_{6}+3 \mathrm{H}_{2} \mathrm{O}\right)$.

In Fig. 5 are given the emission spectra of diaspore, curves $a$ and $b$, temperatures $500^{\circ}$ to $600^{\circ}$ and of hydrotalcite, curve $c$, and temperature $400^{\circ}$. The spectra have no marked bands, except diaspore at $2.78 \mu$.

CYANITE $\left(\mathrm{Al}_{2} \mathrm{SiO}_{5}\right)$.

In Fig. 6, are given the spectral emissivity curves of cyanite (powder, on heater) the estimated temperatures varying from $500^{\circ}$, curve $a$, to $900^{\circ}$, curve $c$ (not drawn to same scale). In this substance, the spectrum of alumina seems to predominate, in marked contrast with the preceding.

$\operatorname{ZIRCON}\left(\mathrm{ZrSiO}_{4}\right)$.

Two spectral energy curves of powdered (brown color) zircon are given in Fig. 7, the temperatures being perhaps $600^{\circ}$ and $700^{\circ}$, respectively. The scale of ordinates of curve $a$ are twice those of curve $b$. Numerous small emission bands are noticeable, which are somewhat shifted from the absorption bands of a transparent plate, having maxima at $2 . \mathrm{I} \mu$, 3.I $\mu$, and $3.6 \mu$, respectively.

TOPAZ $\left[(\mathrm{AlF})_{2} \mathrm{SiO}_{4}\right]$.

In Fig. 8 are given the energy curves of powdered topaz on a heater tube, the temperatures ranging from perhaps $500^{\circ}$ to $900^{\circ}$, curves $a$ to $c$. In this illustration the scale of curve $a=3.6 b$ $=6.7 \mathrm{c}$. The examination was made to compare with previous curves $^{7}$ of a rod of topaz made in an oxyhydrogen flame, hence probably decomposed. Although the temperature of the powder was from $500^{\circ}$ to $600^{\circ}$ lower than that of the rod previously examined, the spectral region with an intense sharp maximum at $2.8 \mu$ is higher than the region at $4.5 \mu$, which is just the opposite of the radiation curves of the rod. This is strong evidence that in the rod the mineral was decomposed into the constituent oxides previously examined. In Fig. 9 are given the spectral radiation curves of an electrically heated rod made of alumina and about I per cent feldspar, curve $a$, and a rod of alumina and I per cent 

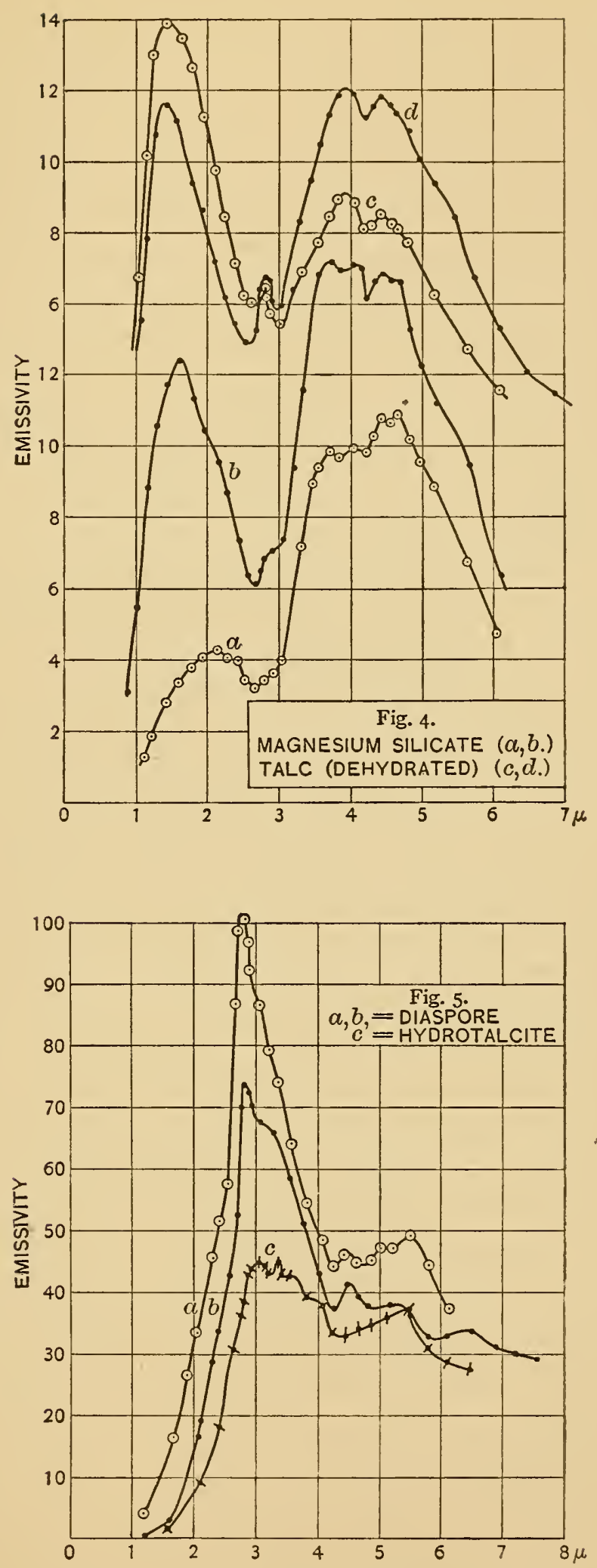
silica, curve $b$. The radiation curves are very similar to those obtained on the decomposed topaz, and seem to indicate a solid solution instead of a true chemical compound; for the spectra appear to be the composite of those of the individual oxides, while in a true compound the individuality of the spectra of the constituents is destroyed and an entirely new spectrum is formed. An excellent example of the latter is zircon, Fig. 7, which has an entirely different spectrum from the pure oxides. Our knowledge of the constitution of mixtures of oxides having high melting points is extremely limited. Whether mixtures of these oxides form a definite compound or simply a solid solution is a mooted question. To melt them in a crucible and keep them pure is a difficult undertaking. The methods of analysis here employed may therefore be of use in furnishing qualitative information as to the physical condition of mixtures of the highly infusible oxides.

\section{RADIATION FROM METALS.}

The question of the luminous efficiency of incandescent filaments has occupied the attention of experimenters from the earliest work on the so-called glow lamp. Evans ${ }^{8}$ described comparisons of the radiation from bright and from black incandescent lamp filaments of carbon, in which he demonstrated very clearly the superiority of the former as a source of light. He selected for this purpose two filaments of similar size and structure, upon one of which he deposited a silver-gray coating of carbon and upon the other a hard coating of carbon of the color of lamp black. He showed that for the same energy input the bright filament emitted the more light. The lamp bulbs containing the black filaments were found much hotter than those containing the silver-gray filaments. He remarks: "I have little doubt that the loss of efficiency when black was due to the energy supplied being radiated in large quantities as heat waves from the blackened surfaces, which these surfaces when bright would not emit." Weber ${ }^{9}$ showed that the total radiation of the untreated carbon was very much greater than the flashed when operated

\footnotetext{
${ }^{8}$ Evans, Proc. Roy. Soc.; Feb. 18, 1886.

${ }^{9}$ Weber, Phys. Rev., 2, pp. Ir 2 and I97; I 894.
} 

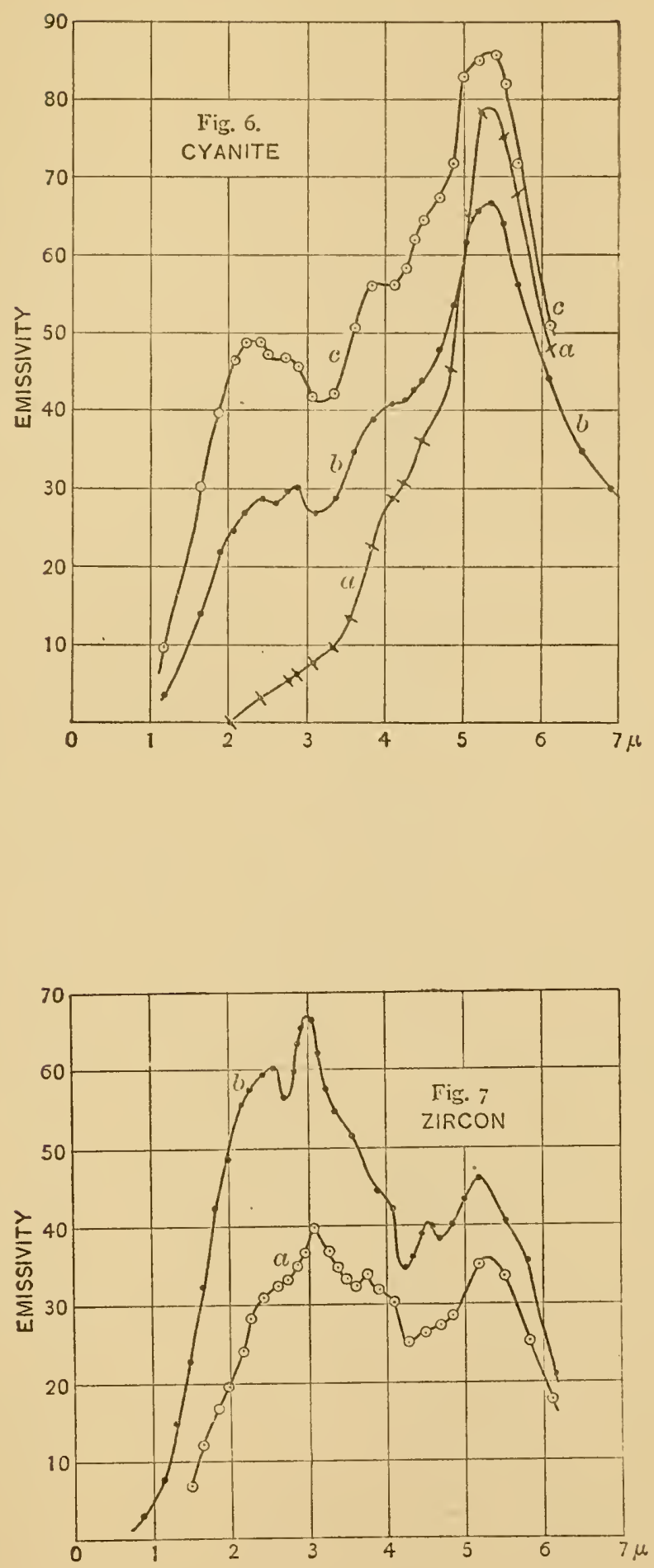
310

Bulletin of the Bureau of Standards.

[Vol. 6, No. 3 .

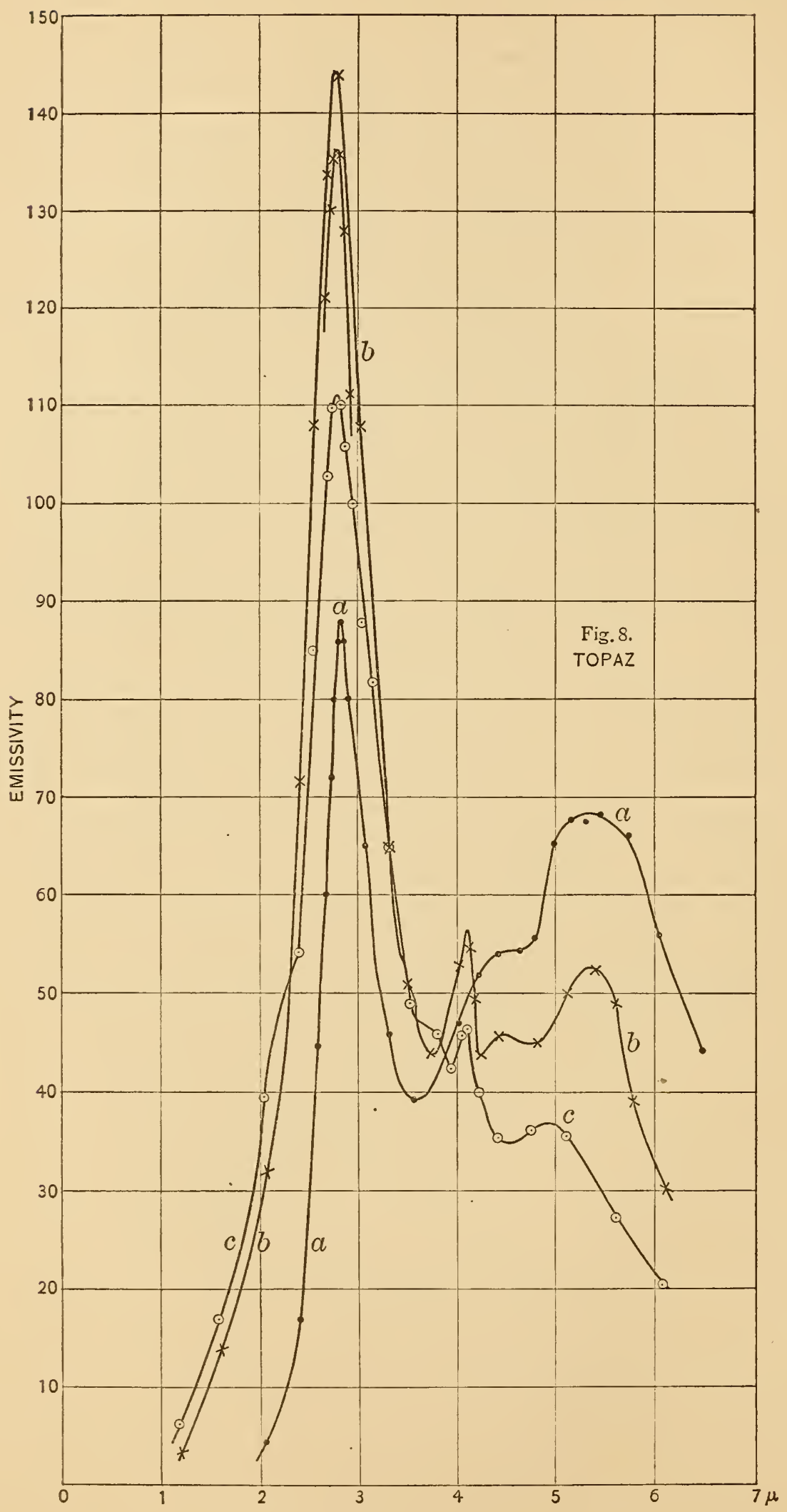




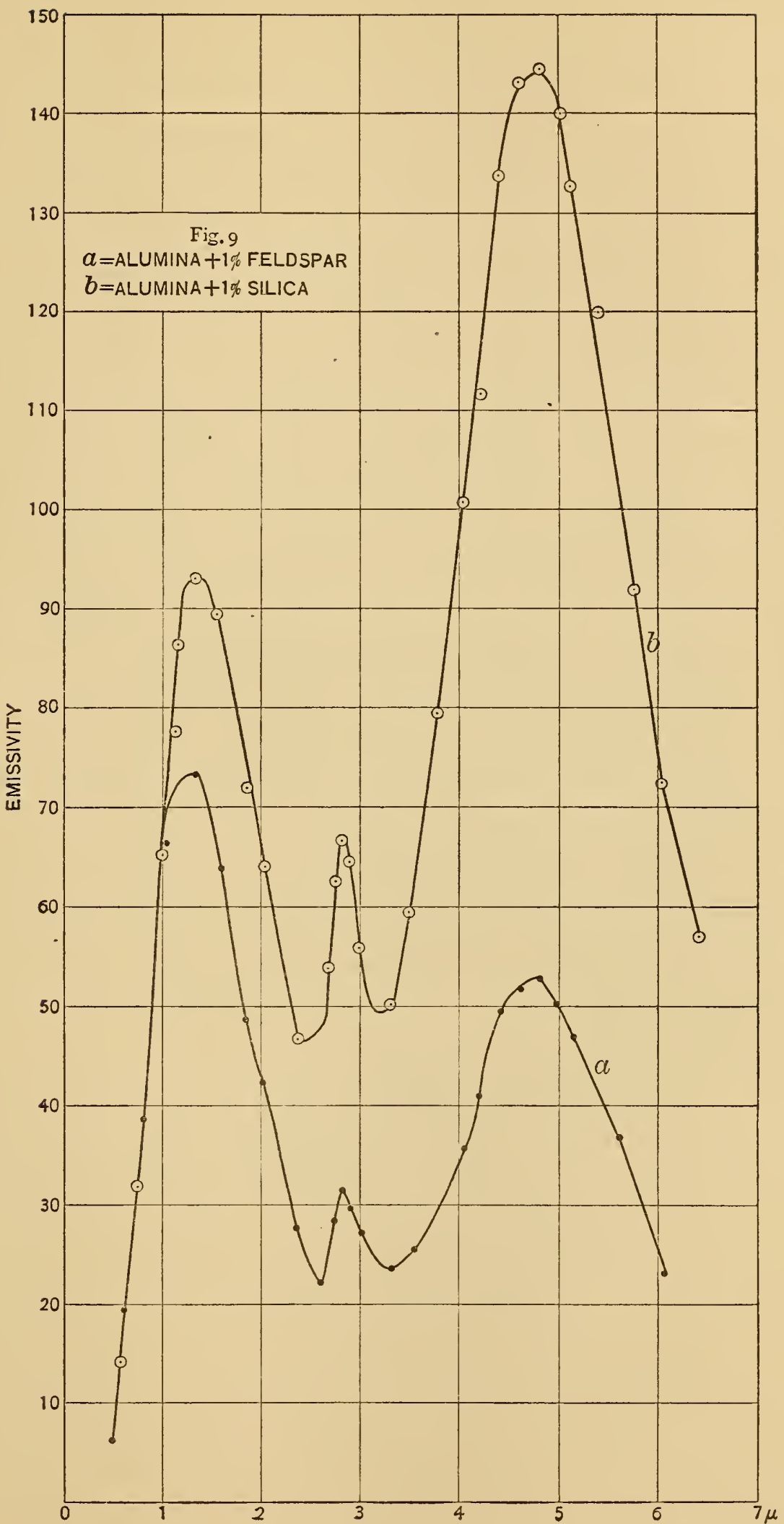


under similar conditions. These observations were verified by Nichols, ${ }^{10}$ who investigated the distribution of energy in the spectra of two lamp filaments similar in every respect except that, after they had been flashed, the one was given a thin coating of carbon by smoking. The lamps could be brought to incandescence separately, but were sealed together in such a manner that whatever changes in vacuum occurred in the one would be shared by the other lamp. He found that the untreated filament emitted much more energy in the infra-red than did the treated filament when operated on the same energy input. Continuing this inquiry as a side issue to the main work on the radiation constants of metals, the writer ${ }^{11}$ published a series of spectral energy curves in which the various filaments are set to the same emissivity at a given wave length, which was taken at $0.7 \mu$. The results having been given considerable publicity and the "color match" 12 method of making some of the comparisons being open to criticism, because the eye is lacking in sensitiveness in the red where radiometrically the energy already assumes large values, it was deemed of importance to repeat the work in order to show the defects in making comparisons which depend upon physiological stimulus to the eye.

The bolometric method of comparing spectral energy was described in this Bulletin, volume 5, page 358. Since the emissivity of the filaments is different, it would be difficult to obtain an

\footnotetext{
${ }^{10}$ Nichols, E. L., Phys. Rev., 2, p. 260; 1894.

11 This Bulletin, 5, p. 339 .

12 The method of comparison of light sources by matching them in color with a photometer sensitive to color differences was described by Nichols in 1894 (Phys. Rev., vol. 2, p. I66), and the present variation in the method as applied to radiators in general is objectionable, because it is not admissible to apply a "color match" or even a bolometric test to one part of the spectrum and then attempt to draw conclusions as to what occurs in other spectral regions. Experience has taught radiometricians that the only way to be certain of one's results is to examine the spectrum from wave length to wave length throughout the whole range. As applied to incandescent lamps (Hyde, Cady, and Middlekauff, Elect. World, 53, 1909; Ill. Eng., Mar., 1909) there is no serious objection, because the energy supplied is dissipated in three important ways, viz, by convection, by conduction (through the lead wires), and by radiation, and since the first two losses can not explain the difference in light efficiency of two lamps, set to a color match, about the only conclusion one can arrive at is that the lamp requiring the greater energy input loses the more by radiation in the infra-red.
} 
intensity match bolometrically at a given wave length without varying the area of the prism face exposed to radiation from different filaments. Not knowing the emissivity, this would be an unsatisfactory method. A spectrophotometer or optical pyrometer should therefore be employed to set the lamps to the same emissivity at, for example, $0.6 \mu$. Since the eye is the most sensitive for the region at about $0.55 \mu$, where the spectral energy is very weak, it was deemed sufficiently accurate to rate the lamps, as was previously done, by matching them in color and then compare the ratios of intensities bolometrically in different parts of the spectrum. If the color match indicates the true energy distribution, then the ratios of the emissivities of the two filaments should be the same throughout the visible spectrum. With an improved and highly sensitive bolometer it was possible to explore the visible spectrum, which was not done in the previous work, and in three series of observations, as will be shown presently, the ratios indicate (from their rapid increase in value) that at $0.75 \mu$ the eye is not able to detect a difference in intensity amounting to about 5 per cent. This would seem to indicate that in addition to being mere guesswork in predicting conditions in the infra-red (since it can not show the manner in which the energy is distributed) the color match is not very applicable to the visible spectrum. As applied to incandescent lamps, it is less hazardous to use the color match in predicting the infra-red emissivity of various lamps, because of what is known from other investigations. Without a wattmeter (or calorimeter), however, the color match tells us nothing, and with it matters are just about where they were before as regards our knowledge of the emission of incandescent filaments in the infra-red.

The majority of the metals have about the same reflectivity in the infra-red, beyond $\mathrm{I} .5 \mu$, hence their emissivity must be about the same, in the infra-red, at the same temperature. On the other hand, their reflectivity is very different in the visible spectrum, and consequently their emissivity must be very different in this region of the spectrum when operated at the same temperature. Hence, on a color match, two metals are not very likely to be at the same temperature. The difference in emissivity in the infra-red, of two metals operated on a color match, will depend principally r $3260-09-2$ 
upon the difference in their reflectivity in the visible spectrum. It might, therefore, have been better to set the two filaments to the same emissivity bolometrically, in the infra-red, and observe their difference in emissivity in the visible spectrum, instead of the reverse process herewith presented. The filaments (if metals) would then be operating at more nearly the same temperature than on a color match. The luminous efficiency would then be obtained at more nearly the same temperature, which is an important desideratum. Instead of a spectrobolometer for making this intensity match in the infra-red it may be possible to use a screen which has a narrow transmission or reflection band, in the infra-red, but opaque to the visible spectrum.

In view of the great importance attributed to the so-called selective emission of the metal filament lamps in the infra-red it is worth while to discuss some recent data on this subject before describing the present results. Nyswander ${ }^{13}$ has published a series of spectral energy curves of an incandescent tungsten filament which apparently indicates bands of selective emission in the infrared, but which in reality are due to atmospheric absorption bands, and also to probable inaccuracies in the so-called slit width correction which is difficult to determine at $\mathrm{I} .7 \mu$, in fluorite prisms. In his earlier work the writer experienced the same difficulties, but after eliminating them, the spectral energy curves of a uniformly heated cavity, as well as those of metal filaments, were always found to be smooth and continuous except at $3 . \mathrm{I} \mu$, which band seems to be due to atmospheric oxygen. From this and from our knowledge of the reflectivity of metals it seems quite certain that in pure metals the spectral radiation curves do not show indentations due to bands of selective emission. Instead of indentations; the emissivity of the metal filaments is greatly and uniformly suppressed in the infra-red, as compared with a complete radiator. (See fig. Io-incorrectly drawn at $0.7 \mu$.)

In the present investigation the carbon and tungsten lamps were matched in color by several observers in the photometry section of this Bureau, and hence considerable accuracy can be claimed for it. The treated carbon filament was of the single loop type while the tungsten filament was a single loop "series lamp." To 
attain greater accuracy straight filaments should be used. The adjustments and procedure in the experiments was the same as in the previous paper. The only changes introduced was a more sensitive bolometer; and the prism was covered with a diaphragm

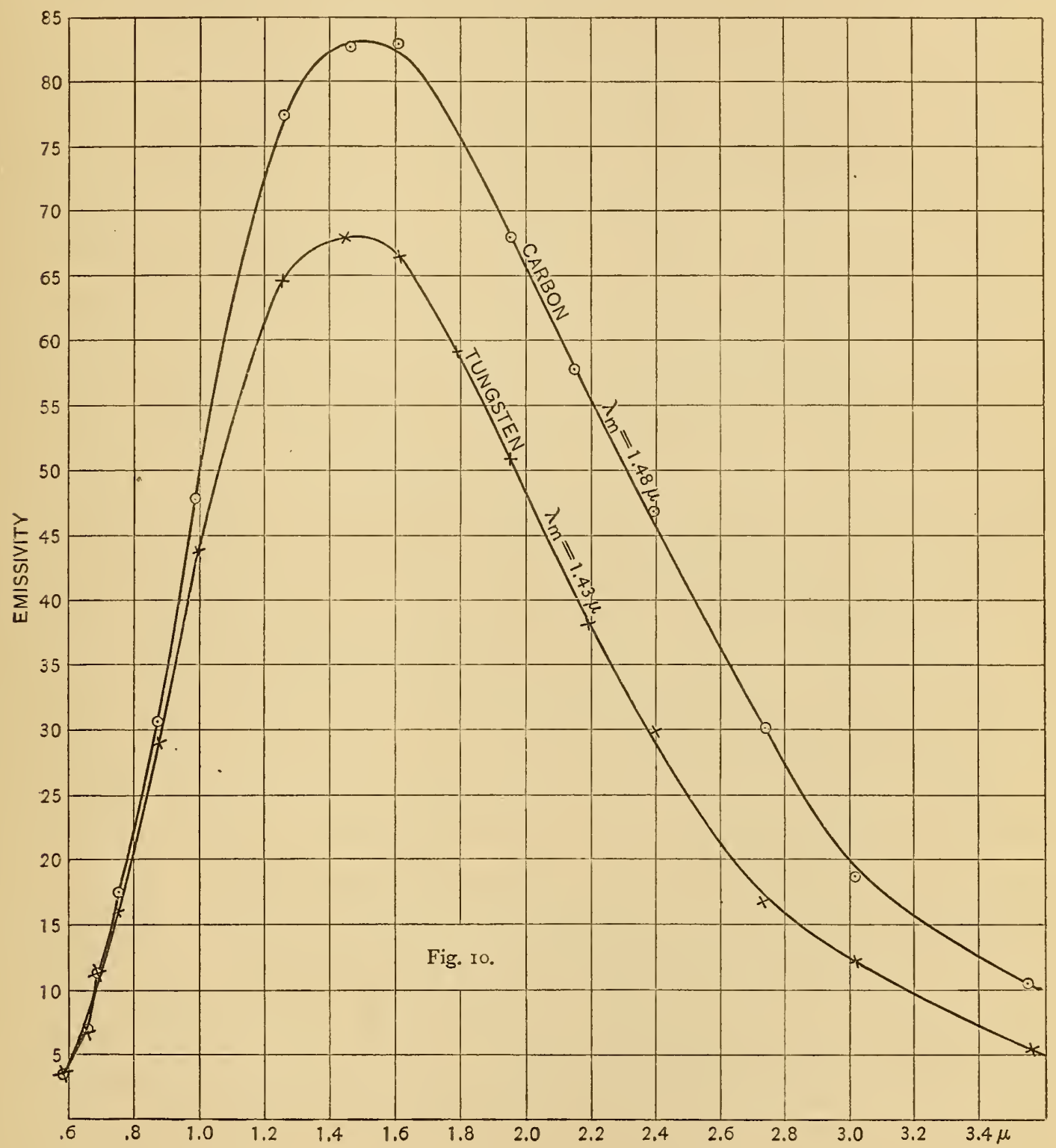

having an opening $8 . \mathrm{mm}$ wide to insure that, whatever the adjustments in the lamps, the same area of prism face was covered by the two filaments. In this manner it was hoped that in varying the adjustments, the same ratios of intensities would be maintained after correcting for variation in sensibility of the bolometer. But 
the curvature of the carbon filament did not admit uniform illumination of the prism face, so that while the ratios for various adjustments gave parallel curves (Fig. I I, $a$ and $b$ ) an additional factor would have to be introduced in order to superpose the curves. This, however, has nothing to do with the present question of the rapid increase in the ratios as we leave the visible spectrum.

In Fig. Io are given the spectral distribution of energy of a treated carbon and a "series" tungsten lamp having filaments about the same diameter. The wave-length of maximum emission of the carbon lamp was $\lambda$ max $=\mathrm{I} .48 \mu$ a $\mathrm{d}$ of the tungsten lamp it was $\lambda \max =1.43 \mu$. Beyond $2.5 \mu$ the curves are not com-

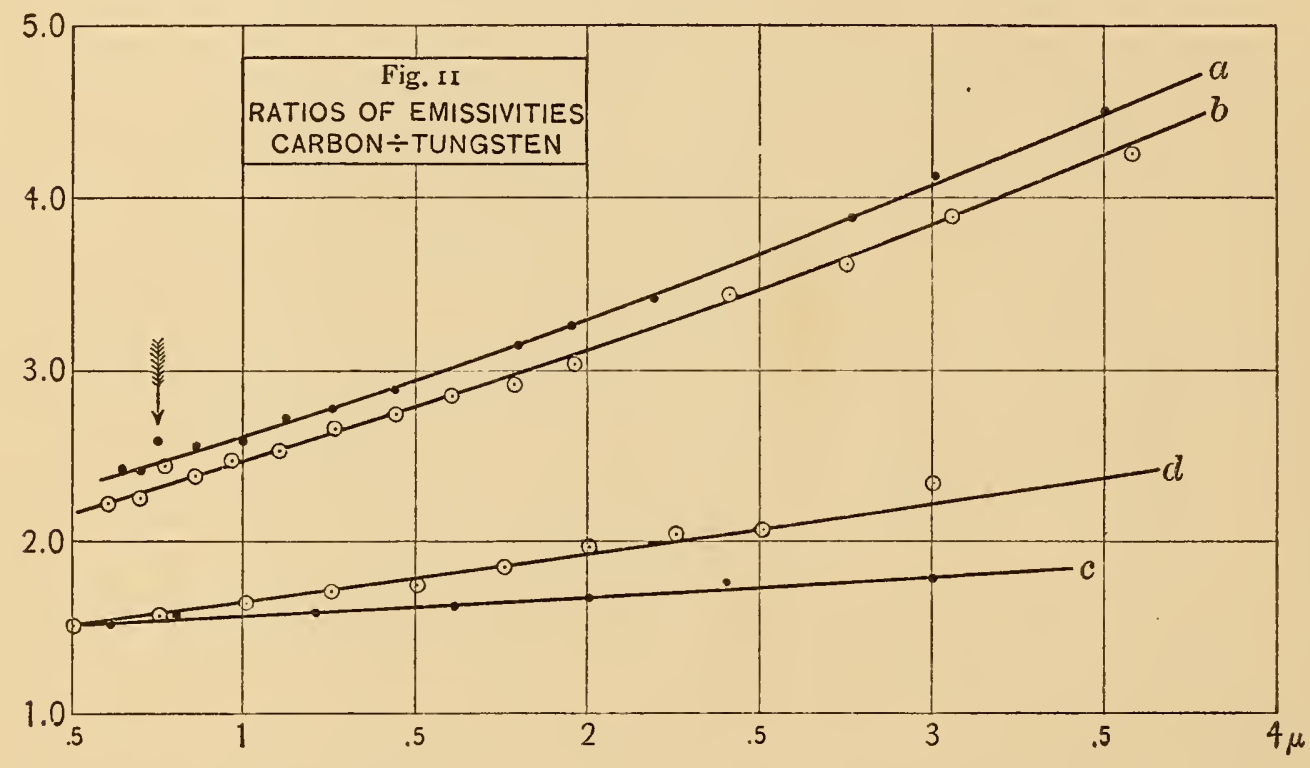

parable on account of the absorption of the glass bulb. The observations on the tungsten lamp were multiplied by a common factor which reduced the intensities to equality at $0.58 \mu$. Here the deflections are small, and while the errors of observations are large, the effect is insignificant in the energy curve. At $0.75 \mu$ the energy curves already depart from equality by about 5 per cent. Here the errors of observation are small because of the large deflections which are at least 5 times those at $0.58 \mu$. In three series of measurements made on different carbon lamps, the energy curves showed an abnormal rise in value at $0.75 \mu$. This rise in emissivity is similar to the spectrophotometric observations of Nichols, ${ }^{14}$ but 
whether it is a reality would require further investigation. This should be done, using a larger dispersion, since the radiometric observations can be made with considerable accuracy in this region, where the spectrophotometer becomes untrustworthy.

In Fig. I I are given the observed ratios of the emissivities of these two lamps. It will be noticed that at $0.75 \mu$ the ratios deviate from the general series by a greater amount than can be attributed to experimental error. Curves $a$ and $b$, Fig. I I, give the ratios of the emissivities (carbon $\div$ tungsten) for a color match when the carbon lamp was operated on about 4.6 w. p. c. (lamp stationary; plane of the filament perpendicular to photometer); curves $c$ and $d$ are similar observations made at an earlier date, when the color match was less accurately determined and the energy input was different. Curves $a$ and $b$ are parallel, as they should be, since all the conditions remained the same except that the lamps were readjusted before the slit in the two series. That these two curves do not coincide is due to lack of uniform illumination of the prism in the two series, as explained on a previous page. If the color match gives us an accurate knowledge of existing conditions, then the bolometrically observed radiation curves of the visible spectrum should be parallel, but not necessarily of equal intensity. The ratio of intensities should therefore be the same throughout the visible spectrum, i. e., the curves should not slope. The curves herewith presented demonstrate conclusively that for a color match (and intensity match at $0.52 \mu$ ) the bolometer shows a difference amounting to 3 to 5 per cent at 0.70 to $0.75 \mu$. The farther the observations extend into the infra-red the greater the ratio of emissivities (carbon $\div$ tungsten), and whatever doubts we have regarding the accuracy of the observations in the visible spectrum, from the observations beyond $0.8 \mu$ there can be no doubt that the eye is incapable of distinguishing variations in emissivity in the deep red, when two lamps are apparently matched in color.

As a finale to this discussion of the inapplicability of the color match (or for that matter any other device than a spectroradiometer) for predicting what happens in one part of the spectrum from observed conditions in another region, let us select the Welsbach mantle and also the same material formed into an electrically heated rod. Let us suppose that it is difficult to estimate 
the efficiency of the mantle; we therefore select the electrically heated rod and determine the watts per candle. Assuming that the emissivity of the oxides is dominated by surface conditions, as in metals (in which the absorption coefficient is extremely large as compared with oxides), we decide from our observations on the electrically heated rod that the same relation of watts per candle must hold in the mantle. We might even conclude that, on account of the cellular structure of the mantle, it is "blacker" than the smooth rod, and hence is less efficient as a source of light.

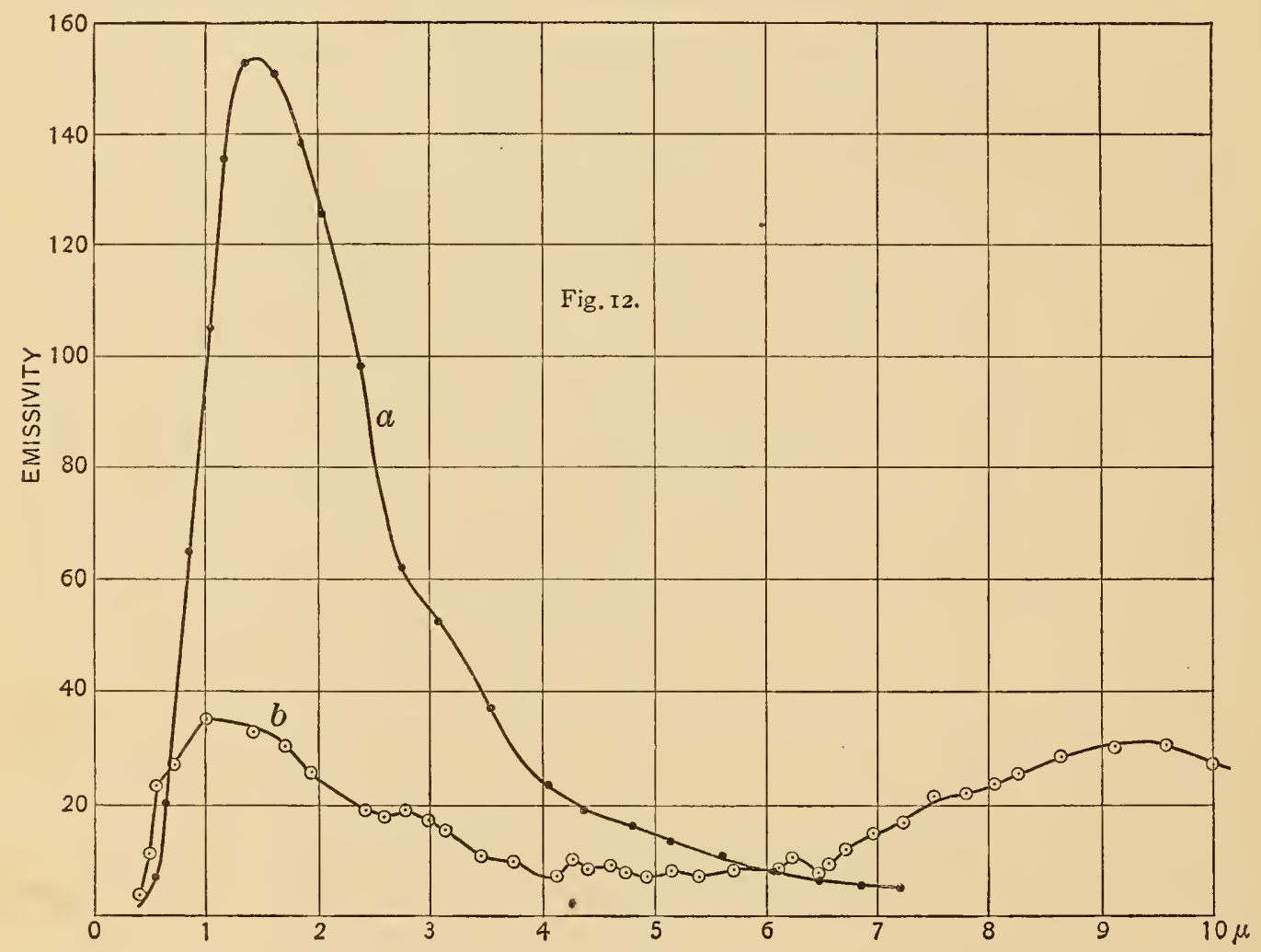

To what absurdities such assumptions can lead will be noticed in Fig. I2, which shows the distribution of energy in the spectrum of the Welsbach mantle, curve $b$ (data from Rubens), and of the same material (99 p. c. thoria I p. c. ceria) in the form of a rod heated electrically. In the latter, curve $a$, Fig. I2, the vertical scale is one-third that of the mantle. It will be observed that the mantle is almost lacking in infra-red radiation. The reason for this is not difficult to find. The oxides are "transparent media," and hence the emissivity is a function of the thickness rather than of the reflecting power (high absorption coefficient), as in metals. The 
small amount of cerium-oxide, which has a high absorption for all the visible spectrum except the yellow and red (combined with a high temperature), is sufficient to produce a saturated radiation in the region of short wave-lengths, but in the infra-red the emissivity is low, due to the lack of thickness of the mantle. On the other hand, the (electrically) heated rod is so thick ( $1.2 \mathrm{~mm}$ in this experiment) that, although the absorption coefficient is low beyond $\mathrm{r} \mu$, the emissivity is high, due to the thickness of the radiating layer.

Washington, August I4, 1909. 